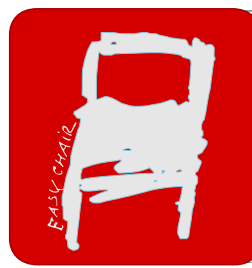

EPiC Series in Health Sciences

Volume 3, 2019, Pages 297-300

CAOS 2019. The 19th Annual Meeting of the International Society for Computer Assisted Orthopaedic Surgery

\title{
Preliminary Planning for a Multi-institutional Database for Ultrasound Bone Segmentation
}

\author{
Prashant Pandey ${ }^{1}$, Hridayi Patel ${ }^{2}$, Pierre Guy ${ }^{3}$, Ilker Hacihaliloglu² , and \\ Antony J. Hodgson ${ }^{4}$ \\ 1 Electrical and Computer Engineering, University of British Columbia Vancouver, BC, Canada \\ prashant@ece.ubc.ca \\ 2 Department of Biomedical Engineering, Rutgers University, Piscataway, NJ, USA \\ 3 Department of Orthopaedics, University of British Columbia, Vancouver, BC, Canada \\ 4 Department of Mechanical Engineering, University of British Columbia, Vancouver, BC, Canada
}

\begin{abstract}
Ultrasound (US) bone segmentation is a key component in many US-based computer assisted orthopaedic systems. Although numerous US bone segmentations techniques exist, there remains no direct way of comparing their performances. This is primarily due to the lack of an accessible US bone image database, and secondly due to a lack of standard validation practices. To address this issue, we are beginning a multi-institutional international collaboration across multiple research centres with the aim of creating an open database for US bone segmentation consisting of several thousand US images and corresponding bone surface segmentations. Our collaboration also aims to address outstanding issues in US bone segmentation, such as determining the reliability of manual segmentations and establishing a set of evaluation metrics which should be reported in future segmentation studies. Finally, we strongly encourage interested researchers to join and contribute to this project as this will help to create a more diverse database and knowledgeable collaboration.
\end{abstract}

\section{Introduction}

Automatic, accurate, and real-time bone surface segmentation in intraoperative ultrasound (US) is a vital part of any ultrasound-guided computer assisted orthopaedic surgery (CAOS) system. Various research groups have developed different bone segmentation methods based on traditional image processing or machine learning-based methods [2]. Validating proposed methods involves comparing the automatically extracted bone surfaces against manual segmentations, often considered the appropriate reference standard and performed by expert radiologist or ultrasound technicians, or against corresponding bone surfaces extract from computed tomography $(\mathrm{CT})$ scans which requires registering the datasets [3].

However, despite the wealth of US bone segmentation techniques, there is no accessible standardized database on which to assess segmentation performance. This makes it challenging to directly compare algorithms, and thus understand which segmentation techniques are most suitable for given clinical requirements [6]. Therefore, we have initiated discussions with 
multiple international research groups with the aim of creating an open database containing US bone images and corresponding bone surface segmentations. A secondary aim of this collaboration is to determine evaluation criteria and validation practices which accurately capture and represent segmentation performance in a repeatable manner. This, for example, includes determining recommended performance metrics when validating techniques. A further aim of this collaboration will be to use the curated dataset to structure and host a segmentation 'challenge', so that existing and new bone segmentation methods' performances can be directly evaluated, compared and reported.

\section{Call for Collaboration}

To date, we have identified collaborators from seven different institutions who will be contributing US images and corresponding bone images to the open database. However, this is an ongoing project and we wish to invite other researchers who are interested in collaborating and contributing to the field of US bone segmentation. Researchers can contribute by sharing any US bone images they have collected as part of previous studies, or plan to collect in future studies, as well as the corresponding manual bone surface segmentations or corresponding CT bone surfaces. Once the database has been published, researchers can also contribute by downloading the US images and submitting independent manual segmentations of the bone surfaces. This will help establish the inter-rater variability in manual US bone segmentation. It is important to note that this is intended to be a continuously evolving, and researchers will be encouraged to continue contributing new images as they are acquired.

\section{Data}

To date, we estimate that over 10,000 US images will be forming the initial database. This will include 2D images, 2D tracked images, and 3D volumes, acquired from either human volunteers or custom phantoms. The images will include scans of the femur, pelvis, spine, humerus, radius, ulna, fibula and tibia, and images will be paired either with corresponding manual bone segmentations or with a corresponding (aligned) bone surface from a CT scan of the subject.

\section{Planned Discussions and Work}

The project aims to address many of the current issues in US bone segmentation. Primarily, the open database can facilitate standardized comparisons of proposed (semi-)automatic bone segmentation techniques. However, other issues include determining the inherent (un)reliability in manual segmentations, which are often used as the 'ground truth' for bone segmentation. Furthermore, we also plan to determine the most suitable validation metrics to use when evaluating bone segmentation performance. Over the years, researchers have proposed a variety of distance metrics (such as mean Euclidean distance, root-mean-square distance error, and Hausdorff distance) [7], pixel classification metrics (such as recall, precision, and F1 score) [1], as well as other clinically-specific or unique metrics. Each metric reflects different aspects of bone segmentation, and we plan to discuss the minimum set of metrics which should be reported in future studies such that direct comparison of accuracies between algorithms is possible. Methods.

We will also discuss using the database in designing more robust machine learning-based segmentation models, such as those published in $[7,1,8]$. In particular, an open database 
containing several thousand US images sourced from multiple subjects, raters, anatomies and US machines captures the natural variety of US patient images, and thus can be used to train a model robust to heterogeneous images. However, it will be necessary to decide which images and what proportion of images should be split into training, validation and test sets, to ensure adequate learning and fair testing. Similarly, we plan to discuss using the database to evaluate the effect of segmentation models on downstream registration, as this is also a key component in US-based CAOS systems.

\section{Conclusion}

We believe that providing an open database for US bone segmentation will benefit the researchers in the field of ultrasound-based surgical interventions. In particular, the database and resulting collaboration will establish a means of standardized comparison between several existing and future segmentation methods. Such an approach has been instrumental in facilitating comparison and development of new segmentation methods in other medical imaging applications [5, 4], and we hope that this database will have a similar effect in US bone segmentation. We encourage all interested researchers to join this collaboration and help build the open database for US bone segmentation.

\section{Acknowledgements}

We would like to thank the current participants of this collaboration: Fernando Arámbula-Cosío (Universidad Nacional Autónoma de México), Guillaume Dardenne (Université de Bretagne Occidentale), Zian Fanti (Universidad Nacional Autónoma de México), Gabor Fichtinger (Queen's University), Parvin Mousavi (Queen's University), Eric Stindel (Université de Bretagne Occidentale), Tamas Ungi (Queen's University), Theo van Walsum (Erasmus University Medical Center), and Wolfgang Wein (ImFusion and Technische Universität München).

\section{References}

[1] Nora Baka, Sieger Leenstra, and Theo van Walsum. Ultrasound aided vertebral level localization for lumbar surgery. IEEE Trans. Med. Imaging, 36(10):1-1, 2017.

[2] Ilker Hacihaliloglu. Ultrasound imaging and segmentation of bone surfaces: A review. TECHNOL$O G Y, 05(02): 74-80$, jun 2017.

[3] Ilker Hacihaliloglu. 3D Ultrasound for Orthopedic Interventions. In Intelligent Orthopaedics, pages 113-129. Springer, Singapore, 2018.

[4] Geert Litjens, Robert Toth, Wendy van de Ven, Caroline Hoeks, Sjoerd Kerkstra, Bram van Ginneken, Graham Vincent, Gwenael Guillard, Neil Birbeck, Jindang Zhang, Robin Strand, Filip Malmberg, Yangming Ou, Christos Davatzikos, Matthias Kirschner, Florian Jung, Jing Yuan, Wu Qiu, Qinquan Gao, Philip "Eddie" Edwards, Bianca Maan, Ferdinand van der Heijden, Soumya Ghose, Jhimli Mitra, Jason Dowling, Dean Barratt, Henkjan Huisman, and Anant Madabhushi. Evaluation of prostate segmentation algorithms for MRI: The PROMISE12 challenge. Med. Image Anal., 18(2):359-373, feb 2014.

[5] Bjoern H. Menze et al. The Multimodal Brain Tumor Image Segmentation Benchmark (BRATS). IEEE Trans. Med. Imaging, 34(10):1993-2024, oct 2015.

[6] Prashant Pandey. Real-time ultrasound bone segmentation and robust US-CT registration for surgical navigation of pelvic fractures. University of British Columbia, 2018. 
[7] M. Villa, G. Dardenne, M. Nasan, H. Letissier, C. Hamitouche, and E. Stindel. FCN-based approach for the automatic segmentation of bone surfaces in ultrasound images. Int. J. Comput. Assist. Radiol. Surg., 13(11):1707-1716, nov 2018.

[8] Puyang Wang, Vishal M. Patel, and Ilker Hacihaliloglu. Simultaneous Segmentation and Classification of Bone Surfaces from Ultrasound Using a Multi-feature Guided CNN. In Lect. Notes Comput. Sci. (including Subser. Lect. Notes Artif. Intell. Lect. Notes Bioinformatics), volume 11073 LNCS, pages 134-142. Springer, Cham, sep 2018. 